\title{
A model for the role of gut bacteria in the development of autoimmunity for type 1 diabetes
}

\author{
Austin G. Davis-Richardson ${ }^{1} \cdot$ Eric W. Triplett ${ }^{1}$
}

Received: 5 December 2014 / Accepted: 27 March 2015 / Published online: 10 May 2015

(C) The Author(s) 2015. This article is published with open access at Springerlink.com

\begin{abstract}
Several lines of evidence suggest a role for the gut microbiome in type 1 diabetes. Treating diabetes-prone rodents with probiotics or antibiotics prevents the development of the disorder. Diabetes-prone rodents also have a distinctly different gut microbiome compared with healthy rodents. Recent studies in children with a high genetic risk for type 1 diabetes demonstrate significant differences in the gut microbiome between children who develop autoimmunity for the disease and those who remain healthy. However, the differences in microbiome composition between autoimmune and healthy children are not consistent across all studies because of the strong environmental influences on microbiome composition, particularly diet and geography. Controlling confounding factors of microbiome composition uncovers bacterial associations with disease. For example, in a human cohort from a single Finnish city where geography is confined, a strong association between one dominant bacterial species, Bacteroides dorei, and type 1 diabetes was discovered (Davis-Richardson et al. Front Microbiol 2014;5:678). Beyond this, recent DNA methylation analyses suggest that a thorough epigenetic analysis of the gut microbiome may be warranted. These studies suggest a testable model whereby a diet high in fat and gluten and low in resistant starch may be the primary driver of gut dysbiosis. This dysbiosis may cause a lack of butyrate production by gut bacteria, which, in turn, leads to the development of a permeable gut followed by autoimmunity. The bacterial community responsible for these
\end{abstract}

Eric W. Triplett

ewt@ufl.edu

1 Microbiology and Cell Science Department, Institute of Food and Agricultural Sciences, 1355 Museum Road, PO Box 110700, Gainesville, FL 32611-0700, USA changes in butyrate production may vary around the world, but bacteria of the genus Bacteroides are thought to play a key role.

Keywords Bacteroidetes · DNA methylation - Gut bacteria · Gut microbial community $\cdot$ Review $\cdot$ Short-chain fatty acids
Abbreviations
BB-DP BioBreeding diabetes-prone
BB-DR BioBreeding diabetes-resistant
DIPP Diabetes Prediction and Prevention
SCFA Short-chain fatty acid
SFB Segmented filamentous bacteria
SPF Specific-pathogen-free

\section{Introduction}

The incidence of type 1 diabetes in many developed countries has been increasing at rates faster than can be explained by the known genetic propensity towards the disease [1]. The environmental triggers of this disease have not yet been identified, despite the many efforts to associate diet, vitamin $\mathrm{D}$, viruses and other factors with disease [1]. A leaky gut has been correlated with type 1 diabetes [2], and an aberrant gut microbiome was proposed as the factor that results in a leaky gut followed by altered immune responses leading to disease [3]. This review describes a model for the role of the gut microbiome in type 1 diabetes based on the latest results.

\section{Animal models}

Past work with non-obese diabetic (NOD) mice and BioBreeding diabetes-prone (BB-DP) rats provided the first 
evidence that bacteria may play an important role in the onset of type 1 diabetes. BB-DP rats fed sulfamethoxazole, trimethoprim and colistin sulphate demonstrate a significantly decreased incidence of diabetes compared with controls [4]. A similar increase in disease-free animals vs controls was observed in NOD mice after administration of doxycycline or vancomycin $[5,6]$. Bacteroides were higher in stools from the BB-DP rat while Lactobacillus was higher in the diabetesresistant rat (BB-DR) [7]. Strains of Lactobacillus johnsonii and Lactobacillus reuteri isolated from BB-DP rats prevented and promoted diabetes in BB-DP rats, respectively [8]. The L. johnsonii strain induces a $\mathrm{T}$ helper 17 cell bias in the mesenteric lymph nodes of C57BL/6 mice while the $L$. reuteri strain does not [9].

Although the incidence of insulitis is not significantly different between germ-free and specific-pathogen-free (SPF) NOD mice, germ-free mice develop insulitis earlier than SPF mice $[10,11]$. The presence of segmented filamentous bacteria (SFB) in female NOD mice is correlated with decreased insulitis incidence and with the percentage of IL-17-expressing lymphocytes [12].

Diet is known to significantly affect diabetes incidence in NOD mice. For example, a high-cereal diet increases diabetes incidence in NOD mice, while a high-protein diet reduces risk [13]. Recent studies observed that acidic drinking water also increases diabetes incidence in NOD mice, and this is prevented by inoculating mice with SFB [14].

\section{Humans}

The results of the murine experiments encouraged efforts to determine whether associations between gut bacteria and type 1 diabetes could be discovered in humans. Rodent model investigations suggested that healthy children may have high populations of probiotic-like bacteria such as Lactobacillus, while the gut microbiome of unhealthy children may be dominated by Bacteroides. Perhaps antibiotic or probiotic use early in life could prevent type 1 diabetes, but this requires more study. Analysis of a Finnish dataset revealed no connection between antibiotic use and autoimmunity for type 1 diabetes [15], but larger cohorts may be needed to observe an antibiotic influence on this disease. Early on, a simple picture was expected to emerge by simply studying the stool content of a few human cohorts with individuals at high genetic risk of type 1 diabetes.

At first, with very small human cohorts with only four healthy children and four children with type 1 diabetes autoantibodies from the Diabetes Prediction and Prevention (DIPP) study in Finland, the human situation did appear to be very similar to the murine one $[16,17]$. These studies showed highly significant taxonomic and functional differences between cases and controls prior to autoimmunity for type 1 diabetes. Levels of Bacteroides were higher in children with autoimmunity, and levels of seemingly protective unclassified Firmicutes were higher in healthy children. These results match the findings of the murine experiments except for the fact that the human samples had negligible amounts of Lactobacillus.

Contrasting results were obtained from an investigation of 298 stool samples collected during the first 3 years of age from 22 case and 22 control children enrolled in the German BABYDIET study [18]. Unlike the small Finnish cohort, no significant differences between taxa were observed between cases and controls after correction for false discovery rate. Instead, network analysis showed that bacterial communities in cases were far less strongly correlated with each other at $<6$ months of age and again at about 2 years of age than in controls. However, the results from the Finnish and German cohorts were similar in that the autoimmune bacterial communities were less stable than the healthy communities. The cause for this instability in autoimmune samples remains unknown.

A still larger set of 947 stool samples collected during the DIPP study from 29 cases and 47 controls during the first 4 years of life was examined [15]. All of the children enrolled in the DIPP study are genetically at high risk for type 1 diabetes. Analysis of these samples revealed a very striking result: approximately 8 months prior to the average time of appearance of the first autoantibody in cases, a large increase in one bacterial species, Bacteroides dorei, was observed. In addition, $B$. dorei was by far the most abundant species in autoimmune prone children, with over $20 \%$ of the population represented by this one species - a rate that was more than double the relative abundance of this species in healthy children. This difference was highly statistically significant even after correcting for false discovery rate and was found to occur at about the same time as the introduction of solid food. A few other minor taxonomic differences were also observed, but nothing as striking as the $B$. dorei result.

So why was the $B$. dorei result seen in the large Finnish DIPP cohort but not in the German BABYDIET study? At first glance, it may appear that since the $B$. dorei result in Finland was not reproduced in Germany, it must then be an anomaly. However, there is one very important difference between the designs of these two cohorts that may explain this difference. The samples used in the DIPP study were all from children born in the same hospital, Turku University Hospital [15]. All of the children lived within $80 \mathrm{~km}$ of each other. Thus, many of the known confounders of the microbiome community were controlled; that is, climate, diet, culture, water supply, pollution levels, air quality and medical practices were all very similar for these children.

In contrast, the children in the BABYDIET cohort were from all over Germany, allowing enough variability in the microbiome confounders to affect microbiome composition. In addition, all of the children in the BABYDIET study had 
first-degree relatives with type 1 diabetes, while in DIPP the children were chosen for the study based on their high-risk HLA genotype at birth. These confounders add to the noise of the data and may explain why no significant taxonomic differences were observed between cases and controls. These confounders may have been sufficient to mask any taxonomic differences that might otherwise have been detected in the BABYDIET study but too weak to mask the network differences seen in this cohort [18].

Hence, a working hypothesis is that geography plays a very large role in whether it is possible to observe significant casecontrol differences in any cohort designed to examine associations between the microbiome and disease. From this perspective, the microbiome results from the BABYDIET and DIPP cohorts do not contradict each other but rather help us understand how best to design cohorts for future diseaserelated microbiome studies.

Two other recent studies from Europe and Mexico showed taxonomic differences in the gut microbiome between individuals with type 1 diabetes and individuals without the disease $[19,20]$. In both studies, levels of Bacteroides were significantly higher in those children with type 1 diabetes compared with healthy children. These studies were intended to show the effect of the disease itself as opposed to identifying a trigger for autoimmunity as in the previous studies. The Mexican study was restricted to Sonora state, which borders Arizona, and was limited to 29 children, including eight controls. Based on the findings of these studies and the preautoimmunity cohorts described above, Bacteroides appears to be a major contributor to microbiome dysbiosis both prior to the development of autoimmunity and after disease diagnosis.

There is evidence that the human genome has some control over the taxonomic composition of bacteria in the gut. However, it is not known whether a genetic propensity to type 1 diabetes, as manifested by HLA genotype, affects bacterial gut composition [21]. The studies conducted to date on the relationship between type 1 diabetes autoimmunity and the microbiome have not seen an HLA genotype effect on the microbiome, but these studies did not have enough participants to observe such an effect $[15,18]$.

The hygiene hypothesis suggests that a lack of exposure to microbes early in life under hygiene conditions in the developed world contributes to a weakened immune system incapable of warding off the effects of detrimental bacteria in the gut [22]. Efforts are underway to examine this issue carefully between Finland and neighbouring Estonia and Russian Karelia [22]. Testing this hypothesis will be difficult in any study protocol. Our view that separating signal from noise is best done by careful cohort design intended to reduce the confounders of the microbiome.

\section{Approaches to reduce these confounders in cohort design}

Our recent results suggest that striking case-control differences can be observed when the geographic distribution of a high-risk cohort is limited to one city and its immediate surroundings. Another issue is whether type 1 diabetes risk is higher in urban or rural areas [23]. Differences in large gut microbiome taxa and alpha diversity differences were recently observed across six clinical sites in children at high genetic risk for type 1 diabetes [24]. Higher socioeconomic status is typically associated with type 1 diabetes and other autoimmune diseases [25]. To date there are no reports in the literature on socioeconomic status and gut microbiome composition, but socioeconomic status has been shown to be associated with the oral microbiome [26]. However, given that geography and diet are well known to affect gut microbiome composition and that socioeconomic status affects diet and place of residence, cohort design should take these factors into account or in some way control for them. To date, no clear associations between type 1 diabetes and diet have been made, but this is being carefully examined by The Environmental Determinants of Diabetes in the Young (TEDDY) study group [27].

An ideal cohort design might be one that includes samples from a set of cities similar to Turku where the cohort includes children all born in the same hospital and all living in the surrounding area. The variability of other environmental factors, such as climate, drinking water, health practices and air quality, are similar across a single city. Having a set of such cities each with children at high risk might allow us to identify bacteria or bacterial functions that are associated with autoimmunity for type 1 diabetes across a broad geographic scale.

Sampling from families may be a means of reducing the noise in the microbiome data. That is, rather than collecting samples from healthy controls matched for genetic risk, control samples could be obtained from healthy siblings. But age matching is important, so noise could be further controlled by enrolling identical and non-identical twins for the study of those at high genetic risk for the disease at birth. This would be a difficult undertaking in a small city such as Turku but might work well in a much larger city in the same country, such as Helsinki.

Another way of reducing noise would be to determine the changes that can occur in the gut microbiome communities in stools in response to long-term exposure to room temperature and air. Approaches must be developed that make it easier for families to collect stools in a manner that allows for rapid freezing. Microbial communities in stools do change significantly after $24 \mathrm{~h}$ of exposure to air, and these changes include a sharp decline in the relative abundance of Bacteroides strains [28], which can survive air but do not divide under aerobic conditions. To date, none of the studies to investigate 
the microbiome of children at high genetic risk for type 1 diabetes has been designed with the microbiome in mind. In addition to the aforementioned improvements in study design and larger cohorts with limited confounding, advancements in big data analysis methods should also help to facilitate withinand between-study analyses.

\section{Does the $B$. dorei genome suggest functions associated with the development of autoimmunity for type 1 diabetes?}

As described above, a recent study showed a strong association between high levels of $B$. dorei in the gut several months prior to the appearance of the first type 1 diabetes autoantibody [15]. A first step towards determining whether $B$. dorei causes type 1 diabetes autoimmunity would be to culture it from the Turku DIPP samples, then inoculate NOD mice with this organism and determine whether this strain(s) reduces the survival rates of the mice. Although this is a high-risk strategy and not without significant intellectual challenges, it would be worthwhile to conduct such an experiment because even though $B$. dorei may not be involved in type 1 diabetes onset in other populations, a certain set of functions expressed by $B$. dorei may have wide applicability to autoimmune disease around the globe.

As more bacterial metagenomes are obtained from stool samples of high-risk children, quantifying the relative abundance of homologues or paralogues of $B$. dorei genes will be an easy task. Additional Bacteroides species may be associated with type 1 diabetes autoimmunity in other cohorts, which will allow us to build a database of genes in common with all Bacteroides associated with diseases vs those genes in Bacteroides species not associated with disease. A similar approach can be used on bacterial species or strains thought to be protective against the disease. Bacterial functions associated with disease have been listed for a very small cohort from the DIPP study [16]. Further studies are warranted on an increased number of individuals and more samples across additional time points.

\section{Is diet the source of high levels of Bacteroides associated with type 1 diabetes autoimmunity?}

As Bacteroides have been associated with type 1 diabetes autoantibodies, the next obvious question pertains to the source of these bacteria. That is, if a single species or set of closely related species is associated with type 1 diabetes, the need to hunt for more bacterial associations with type 1 diabetes declines and the problem becomes one of searching for those environmental factors that increase the abundance of these Bacteroides species in the gut. In the stool samples of autoimmune Turku children, levels of $B$. dorei peak at about 7 months of age, about 1 month prior to the Finnish national standard for the timeframe of solid food introduction [15]. If the early introduction of solid food is related to raised Bacteroides levels, which foods are known to increase Bacteroides abundance in the gut?

High-fat and high-gluten diets encourage colonisation of the gut by Bacteroides. Bacteroides populations in the human gut have been strongly correlated with a diet high in protein, animal fat, cholesterol and other fats [29]. Bacteroides are well known as the primary proteolytic bacteria in the gut [30]. Not surprisingly, vegans and vegetarians have low levels of Bacteroides in their stools [31]. To date, connections between a vegetarian diet and the progression towards type 1 diabetes have not been studied.

Examination of the gut microbiome in coeliac disease suggests that gluten may play a role in Bacteroides abundance. The prevalence of $B$. dorei was $67 \%$ higher in children with active coeliac disease compared with the same children on a gluten-free diet for two years [32]. Similarly, formula-fed children at high risk for coeliac disease were approximately sixtimes more likely to possess $B$. dorei in stool than low-risk controls [33]. These results encourage further work to determine whether gluten increases population growth of $B$. dorei in children at high risk for autoimmunity. Resistant starch may also play a significant role in preventing type 1 diabetes as it has been reported to increase faecal butyrate levels [34].

\section{Association between gut integrity and type 1 diabetes in humans}

The onset of type 1 diabetes might involve an aberrant intestinal microbiome creating the conditions for gut leakiness followed by mucosal immune responses that lead to an attack of insulin-producing islet cells [3]. Consistent with this notion, type 1 diabetic individuals have been shown to have a leakier gut than controls [35-37], and it has been reported that this leakiness is not caused by type 1 diabetes, rather, type 1 diabetes is preceded by a leaky gut [2]. In the NOD mouse model, a leaky gut can lead to the activation of diabetogenic $\mathrm{CD} 8^{+}$ cells which, in turn, lead to the development of insulitis [38]. Mechanisms for a connection between the leaky gut and type 1 diabetes have been reviewed previously [39, 40].

\section{A role for short-chain fatty acids and gut integrity}

Butyrate is one of the short-chain fatty acids (SCFAs) produced by bacterial fermentation in the gut. The ratio of butyrate:acetate:proprionate in the gut varies widely, but these three SCFAs make up the majority of the SCFA content in this organ. The concentration of SCFAs in the gut can be as high as 
$140 \mathrm{mmol} / 1$ and these SCFAs can provide up to $10 \%$ of the daily human caloric needs [41]. Butyrate reduces transmembrane transport of Escherichia coli across confluent monolayers of colon-derived cell lines [42]. In addition, inflammation may reduce butyrate transport through the downregulation of a butyrate transporter [43].

Butyrate is also known to play two important roles in maintaining the integrity of the epithelial layer. First, butyrate induces the expression of mucin-producing genes in the epithelial layer [44]. Mucin is a host glycoprotein that protects the epithelial layer from attack by bacteria or bacterial metabolites. Second, butyrate restores and maintains tight junction assembly in epithelial layers [45, 46]. These tight junctions are formed between epithelial cells and serve as gatekeepers for metabolite transport across the epithelium. Other SCFAs do not induce tight junction formation or mucin synthesis.

Healthy children have a higher proportion of butyrateproducing gut bacteria in their microbiomes compared with children expressing at least one beta islet cell autoantibody $[16,47]$. Based on this, the ratio of butyrate-producing to other SCFA-producing bacteria in the gut was proposed to be a key regulator in determining the gut health of a child at high risk for type 1 diabetes [16]. However, there is no evidence to date linking butyrate levels in the gut to the progression of diabetes in either animals or humans.

\section{Can gut bacteria regulate the epigenome of the human epithelial cell?}

Butyrate has roles other than maintenance of the integrity of the gut epithelial layer. In particular, there is increasing evidence that butyrate has epigenetic effects that may be very important in type 1 diabetes, as unknown environmental triggers must be playing a role in the rapid increase in the incidence of this disease [48].

Butyrate induces the methylation of promoter regions, which causes both up- and downregulation in different sets of human genes [49]. Histone acetylation also appears to be regulated by butyrate production. Butyrate reduces lipopolysaccharide-induced inflammation in the intestine through modulation of antioxidant defence systems [50], nitric oxide production, and expression of inflammatory cytokines [51]. Many of these effects appear to be modulated by the inhibition of histone deacetylases in macrophages [51]. Also, histone acetylation in the promoter of the Foxp3 locus

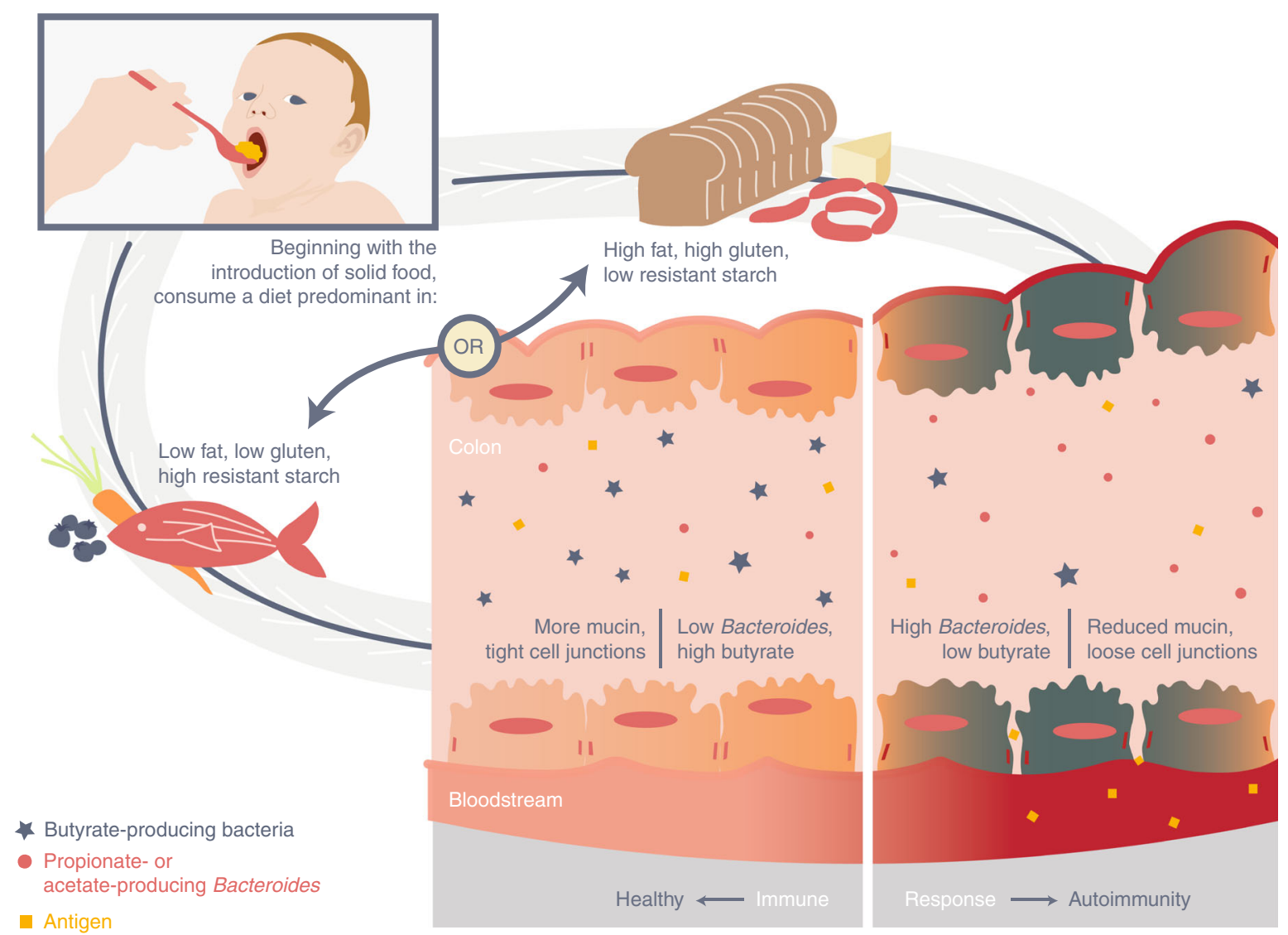

Fig. 1 Model for the development of autoimmunity for type 1 diabetes based on a diet-induced disproportionate high number of Bacteroides in the gut, leading to a leaky gut and autoimmunity for the disease 
induces the differentiation of regulatory $\mathrm{T}$ cells in mice [52]. Several epigenetic changes in host epithelial cells may be caused by increased butyrate production from probiotic strains [53].

\section{Is there a role for the methylome of the gut microbiome in the development of type 1 diabetes autoimmunity?}

The genomes of two of the $B$. dorei strains from the DIPP Turku cohort have been sequenced to completion and, physiologically, appear to be typical Bacteroides in that they metabolise a broad spectrum of compounds and have many antibiotic-resistance genes [54]. One of these genomes was derived from a child who later developed type 1 diabetesassociated autoantibodies (case) while the other genome was from a high-risk healthy child (control). The case genome includes a bacteriophage with a DNA adenine methyltransferase gene that appears to be responsible for the methylation of all but three of the 20,554 GATC motifs in the genome [54]. Such phage-borne DNA methyltransferases have been found in several bacteria [55]. None of the nearly 19,000 GATC motifs in the control genome were methylated, presumably because it lacked this phage and its orphan DNA methyltransferase gene. This work was the first report of DNA methylation differences within a bacterial species in the microbiome [54].

Although these methylation results from two $B$. dorei genomes cannot be associated with disease, they suggest that a wider examination of methylation patterns in more samples is warranted. DNA methylation patterns are known to affect gene expression in bacteria [56]. They control epigenetic responses that are often regulated by environmental exposure. Indeed, a few methylation sites in the human genome have been associated with type 1 diabetes pathogenesis [57, 58]. It is therefore conceivable that methylation differences in the genomes of bacteria associated with type 1 diabetes may play a role in disease.

\section{Conclusions}

The analyses described above suggest a revised model for the role of the microbiome in type 1 diabetes, one that is based on a previous model [16] but takes into account the role of diet (Fig. 1). In this revised model, type 1 diabetes autoimmunity occurs when a high-fat, high-gluten diet, perhaps one with a low level of resistant starch, increases Bacteroides colonisation in the gut. These bacteria ferment glucose to SCFAs other than butyrate. The lack of butyrate production reduces the likelihood of an intact gut epithelial layer. The resulting permeability causes bacterial antigen exposure that induces immune responses leading to type 1 diabetes autoimmunity.
Recent results suggest that the many confounders of gut microbiome composition need to be considered in cohort design for the optimal discovery of associations between the gut microbiome and disease. A cohort carefully designed to control for geography and diet as much as possible can eliminate much of the noise that is often associated with microbiome data. Future work will require experiments or observational studies that directly test each point in the model (Fig. 1).

Acknowledgements The authors apologise to the many authors of original research not cited in this manuscript because of space limitations. The authors thank Mark Atkinson (University of Florida, Gainesville, FL, USA) for encouraging the authors to write and submit this review and for his editorial comments. The authors also thank Stacey Jones of the University of Florida's IFAS Communications for preparing the illustration shown in Fig. 1.

Funding This research was generously supported by two grants from the JDRF (www.jdrf.org) (Projects 17-2011-266 and 2-SRA-2015-308Q-R to EWT).

Duality of interest The authors declare that there is no duality of interest associated with this manuscript.

Contribution statement The authors were responsible for the conception, design and drafting of this manuscript and approved the final version for publication.

Open Access This article is distributed under the terms of the Creative Commons Attribution 4.0 International License (http:// creativecommons.org/licenses/by/4.0/), which permits unrestricted use, distribution, and reproduction in any medium, provided you give appropriate credit to the original author(s) and the source, provide a link to the Creative Commons license, and indicate if changes were made.

\section{References}

1. Atkinson MA, Eisenbarth GS, Michels AW (2014) Type 1 diabetes. Lancet 383:69-82

2. Bosi E, Molteni L, Radaelli M et al (2006) Increased intestinal permeability precedes clinical onset of type 1 diabetes. Diabetologia 49:2824-2827

3. Vaarala O, Atkinson M, Neu J (2008) The "perfect storm" for type 1 diabetes - the complex interplay between intestinal microbiota, gut permeability, and mucosal immunity. Diabetes 57:2555-2562

4. Brugman S, Klatter FA, Visser JT et al (2006) Antibiotic treatment partially protects against type 1 diabetes in the Bio-Breeding diabetes-prone rat. Is the gut flora involved in the development of type 1 diabetes? Diabetologia 49:2105-2108

5. Schwartz RF, Neu J, Schatz D, Atkinson MA, Wasserfall C (2007) Comment on: Brugman $S$ et al. (2006) Antibiotic treatment partially protects against type 1 diabetes in the Bio-Breeding diabetes-prone rat. Is the gut flora involved in the development of type 1 diabetes? Diabetologia 49:2105-2108, Diabetologia 50: 220-221

6. Hansen CHF, Krych L, Nielsen DS et al (2012) Early life treatment with vancomycin propagates Akkermansia muciniphila and reduces diabetes incidence in the NOD mouse. Diabetologia 55:2285-2294

7. Roesch LF, Lorca GL, Casella G et al (2009) Culture-independent identification of gut bacteria correlated with the onset of diabetes in a rat model. ISME J 3:536-548 
8. Valladares R, Sankar D, Li N et al (2010) Lactobacillus johnsonii N6.2 mitigates the development of type 1 diabetes in BB-DP rats. PLoS ONE 5, e10507

9. Lau K, Benitez P, Ardissone A et al (2011) Inhibition of type 1 diabetes correlated to a Lactobacillus johnsonii N6.2-mediated Th17 bias. J Immunol 186:3538-3546

10. Alam C, Bittoun E, Bhagwat D et al (2011) Effects of a germ-free environment on gut immune regulation and diabetes progression in non-obese diabetic (NOD) mice. Diabetologia 54:1398-1406

11. King C, Sarvetnick N (2011) The incidence of type-1 diabetes in NOD mice is modulated by restricted flora not germ-free conditions. PLoS ONE 6, e17049

12. Kriegel MA, Sefik E, Hill JA, Wu H-J, Benoist C, Mathis D (2011) Naturally transmitted segmented filamentous bacteria segregate with diabetes protection in nonobese diabetic mice. Proc Natl Acad Sci U S A 108:11548-11553

13. Patrick C, Wang G-S, Lefebvre DE et al (2013) Promotion of autoimmune diabetes by cereal diet in the presence or absence of microbes associated with gut immune activation, regulatory imbalance, and altered cathelicidin antimicrobial peptide. Diabetes 62:2036-2047

14. Sofi MH, Gudi R, Karumuthil-Melehil S, Perez N, Johnson BM, Vasu C (2014) pH of drinking water influences the composition of gut microbiome and type 1 diabetes incidence. Diabetes 63:632-644

15. Davis-Richardson AG, Ardissone AN, Dias R et al (2014) Bacteroides dorei dominates gut microbiome prior to autoimmunity in Finnish children at high risk for type 1 diabetes. Front Microbiol 5:678

16. Brown CT, Davis-Richardson AG, Giongo A et al (2011) Gut microbiome metagenomics analysis suggests a functional model for the development of autoimmunity for type 1 diabetes. PLoS ONE 6, e25792

17. Giongo A, Gano KA, Crabb DB et al (2011) Toward defining the autoimmune microbiome for type 1 diabetes. ISME J 5:82-91

18. Endesfelder D, zu Castell W, Ardissone A et al (2014) Compromised gut microbiota networks in children with anti-islet cell autoimmunity. Diabetes 63:2006-2014

19. de Goffau MC, Fuentes S, van den Bogert B (2014) Aberrant gut microbiota composition at the onset of type 1 diabetes in young children. Diabetologia 57:1569-1577

20. Mejía-León ME, Petrosino JF, Ajami NJ et al (2014) Fecal microbiota imbalance in Mexican children with type 1 diabetes. Sci Rep $4: 3814$

21. Goodrich JK, Waters JL, Poole AC et al (2014) Host genetics and the gut microbiome can both influence metabolic phenotypes. Cell 159:789-799

22. Kondrashova A, Seiskari T, Ilonen J, Knip M, Hyöty H (2012) The 'Hygiene hypothesis' and the sharp gradient in the incidence of autoimmune and allergic diseases between Russian Karelia and Finland. APMIS 121:478-493

23. Borchers AT, Uibo R, Gershwin ME (2010) The geoepidemiology of type 1 diabetes. Autoimmun Rev 9:A355-A365

24. Kemppainen KM, Ardissone AN, Davis-Richardson AG et al (2015) Early childhood gut microbiomes show strong geographic differences among subjects at high risk for type 1 diabetes. Diabetes Care 38:329-332

25. Calixto 0-J, Anaya J-M (2014) Socioeconomic status. The relationship with health and autoimmune diseases. Autoimmun Rev 13:641-654

26. Belstrom D, Holmstrup $\mathrm{P}$, Nielsen $\mathrm{CH}$ et al (2014) Bacterial profiles of saliva in relation to diet, lifestyle factors, and socioeconomic status. J Oral Microbiol 6:23609

27. Norris JM (2010) Infant and childhood diet and type 1 diabetes risk: recent advances and prospects. Curr Diab Rep 10:345-349
28. Roesch LFW, Casella G, Simell O et al (2009) Influence of fecal sample storage on bacterial community diversity. Open Microbiol J 3:40-46

29. Wu GD, Chen J, Hoffmann C et al (2011) Linking long-term dietary patterns with gut microbial enterotypes. Science 334:105-108

30. Scott K, Gratz S, Sheridan P, Flint H, Duncan S (2013) The influence of diet on the gut microbiota. Pharmacol Res 69:52-60

31. Zimmer J, Lange B, Frick J-S et al (2011) A vegan or vegetarian diet substantially alters the human colonic faecal microbiota. Eur J Clin Nutr 66:53-60

32. Sanchez E, Donat E, Ribes-Koninckx C, Calabuig M, Sanz Y (2010) Intestinal Bacteroides species associated with coeliac disease. J Clin Pathol 63:1105-1111

33. Sanchez E, de Palma G, Capilla A et al (2011) Influence of environmental and genetic factors linked to celiac disease risk on infant gut colonization by Bacteroides species. Appl Environ Microbiol 77:5316-5323

34. McOrist AL, Miller RB, Bird AR et al (2011) Fecal butyrate levels vary widely among individuals but are usually increased by a diet high in resistant starch. J Nutr 141:883-889

35. Kuitunen M, Saukkonen T, Ilonen J, Akerblom HK, Savilahti E (2002) Intestinal permeability to mannitol and lactulose in children with type 1 diabetes with the HLA-DQB $1 * 02$ allele. Autoimmunity 35:365-368

36. Sapone A, de Magistris L, Pietzak M et al (2006) Zonulin upregulation is associated with increased gut permeability in subjects with type 1 diabetes and their relatives. Diabetes 55:1443-1449

37. Secondulfo M, Iafusco D, Carratu R, deMagistris L, Sapone A, Generoso M (2004) Ultrastructural mucosal alterations and increased intestinal permeability in non-celiac, type I diabetic patients. Dig Liver Dis 36:35-45

38. Lee AS, Gibson DL, Zhang Y, Sham HP, Vallance BA, Dutz JP (2010) Gut barrier disruption by an enteric bacterial pathogen accelerates insulitis in NOD mice. Diabetologia 53:741-748

39. de Kort S, Keszthelyi D, Masclee AAM (2011) Leaky gut and diabetes mellitus: what is the link? Obes Rev 12:449-458

40. Vaarala $\mathrm{O}(2008)$ Leaking gut in type 1 diabetes. Curr Opin Gastroenterol 24:701-706

41. Brahe LK, Astrup A, Larsen LH (2013) Is butyrate the link between diet, intestinal microbiota and obesity-related metabolic diseases? Obes Rev 14:950-959

42. Lewis K, Lutgendorff F, Phan V, Soderholm JD, Sherman PM, McKay DM (2010) Stressed epithelia is reduced by butyrate. Inflamm Bowel Dis 16:1138-1148

43. Thibault R, Blachier F, Darcy-Vrillon B, de Coppet P, Bourreille A, Segain J-P (2010) Butyrate utilization by the colonic mucosa in inflammatory bowel diseases: a transport deficiency. Inflamm Bowel Dis 16:684-695

44. Burger-van Paassen N, Vincent A, Pulman PJ et al (2009) The regulation of intestinal mucin MUC2 expression by short chain fatty acids: implications for epithelial protection. Biochem $\mathrm{J}$ 420:211-219

45. Peng L, Li Z-R, Green RS, Holzman IR, Lin J (2009) Butyrate enhances the intestinal barrier by facilitating tight junction assembly via activation of AMP-activated protein kinase in Caco-2 cell monolayers. J Nutr 139:1619-1625

46. Plöger S, Stumpff F, Penner GB et al (2012) Microbial butyrate and its role for barrier function in the gastrointestinal tract. Ann NY Acad Sci 1258:52-59

47. de Goffau MC, Luopajarvi K, Knip M et al (2013) Fecal microbiota composition differs between children with $\beta$-cell autoimmunity and those without. Diabetes 62:1238-1244

48. Paparo L, Di Cotanzo M, Di Scala C et al (2014) The influence of early life nutrition on epigenetic regulatory mechanisms of the immune system. Nutrients 6:4706-4719 
49. Daly K, Shirazi-Beechey SP (2006) Microarray analysis of butyrate regulated genes in colonic epithelial cells. DNA Cell Biol 25:49-62

50. Russo I, Luciani A, de Cicco P, Troncone E, Ciacci C (2012) Butyrate attenuates lipopolysaccharide-induced inflammation in intestinal cells and Crohn's mucosa through modulation of antioxidant defense machinery. PLoS ONE 7, e32841

51. Chang PV, Hao L, Offermanns S, Medzhitov R (2014) The microbial metabolite butyrate regulates intestinal macrophage function via histone deacetylase inhibition. Proc Natl Acad Sci U S A 111:2247-2252

52. Furusawa Y, Obata Y, Fukuda S et al (2013) Commensal microbe-derived butyrate induces the differentiation of colonic regulatory T cells. Nature 504:446-450

53. Licciardi PV, Wong S-S, Tang MLK, Karagiannis TC (2010) Epigenome targeting by probiotic metabolites. Gut Pathog 2:24
54. Leonard MT, Davis-Richardson AG, Ardissone AN et al (2014) The methylome of the gut microbiome: disparate Dam methylation patterns in intestinal Bacteroides dorei. Front Microbiol 5:361

55. Murphy J, Mahony J, Ainsworth S, Nauta A, van Sinderen D (2013) Bacteriophage orphan DNA methyltransferases: insights from their bacterial origin, function, and occurrence. Appl Environ Microbiol 79:7547-7555

56. Marinus MG, Casadeus J (2009) Roles of DNA adenine methylation in host-pathogen interactions: mismatch repair, transcriptional regulation, and more. FEMS Microbiol Rev 33:488-503

57. Rakyan VK, Beyan H, Down TA et al (2011) Identification of type 1 diabetes-associated DNA methylation variable positions that precede disease diagnosis. PLoS Genet 7, e1002300

58. Stefan M, Zhang W, Concepcion E, Yi Z, Tomer Y (2014) DNA methylation profiles in type 1 diabetes twins point to strong epigenetic effects in etiology. J Autoimmun 50:33-37 